\title{
Das Gebet eines Hyperchristen? Zur Dekonstruktion einer religiösen Gattung in Frost von Thomas Bernhard'
}

\begin{abstract}
[Wir] denken, verschweigen aber: wer denkt, löst auf, hebt auf, katastrophiert, demoliert, zersetzt, denn Denken ist folgerichtig die konsequente Auflösung aller Begriffe. ${ }^{2}$
\end{abstract}

Gleich ob Siddur (hebräisch סידור, „Ordnung") im Judentum, Vaterunser im Christentum oder Al-Fātiha (arabisch الفاتحة, »die Eröffnung«) im Islam - die Zentralgebete der drei semitischen Buchreligionen weisen trotz aller Unterscheidungsmerkmale eine funktionale Gemeinsamkeit auf: Sie regulieren die religiöse und kulturelle Integration in Glaubensgemeinschaften und haben somit einen sinn- und identitätssichernden Charakter. Damit das religiöse Weltbild seine erklärende und normative Kraft entfalten kann, wird das Gebet im jeweiligen Kult immer wieder rituell-performativ nachvollzogen. ${ }^{3}$ Innerhalb des Systems Religion müssen alle kanonisierten Gebetstexte als normativ gültige Muster behandelt werden und sind dementsprechend in ihrem Wortlaut und in ihrem Umfang fixiert.

In Anlehnung an Jan Assmanns theoretische Überlegungen zu Kanon und Kommentar ${ }^{4}$ lässt sich aus dem oben angedeuteten Sachverhalt der Normativität eine erste allgemeine Beobachtung zur Funktionsweise von kanonischen Gebeten und Gebetsstrukturen ableiten: nämlich dass über ihre Repetition und Reproduktion `heilige Regeln der Reinheit und Nichtfortschreibbarkeit wachen.

1 In diesem Beitrag werden Teile meines Dissertationsprojektes im Fachbereich Literaturwissenschaft/Germanistik vorgestellt, das ich im Rahmen des Forschungsfeldes »Kulturdynamiken der Religion" am Exzellenzcluster "Kulturelle Grundlagen von Integration" an der Universität Konstanz bearbeite.

2 Thomas Bernhard: Nie und mit nichts fertig werden. Rede anläßlich der Büchner-Preisverleihung. In: Jahrbuch der deutschen Akademie für Sprache und Dichtung. Darmstadt 1970, 83-84, hier 83.

3 Zur weltbildbezogenen und selbstreferentiellen Funktion religiöser Rituale Klaus Bayer: Religiöse Sprache. Thesen zur Einführung. Berlin 2009, besonders 24-34.

4 Vgl. Jan Assmann: Text und Kommentar. Einführung. In: ders. u. Burkhard Gladigow (Hg.): Text und Kommentar. München 1995, 9-34. Zur Theorie der Kanonisierung kultureller Grundtexte s. ebd., $10 \mathrm{ff}$. Zur identitäts- und gemeinschaftsbildenden Funktion des Gebets siehe Albert Gerhards, Andrea Doeker u. Peter Ebenbauer (Hg.): Identität durch Gebet. Zur gemeinschaftsbildenden Funktion institutionalisierten Betens in Judentum und Christentum. Paderborn u. a. 2003. 
Auch ein zweiter für die Stabilität des kanonischen Prekativs ${ }^{5}$ maßgeblicher Faktor ist schnell zu ermitteln. Wie alle normativen systemgebundenen Konstruktionen unterliegt auch der Umgang mit Texten des religiösen Kanons einer strikten Untersagung jeglicher Depragmatisierung und Dekontextualisierung, mit der Konsequenz, dass konträres Handeln als blasphemisch, sakrilegisch oder profanierend verurteilt wird.

Doch in der Literatur der europäischen Moderne lässt sich trotz der Tabuisierung jeglicher "Akkomodation des Textes in Form redaktioneller Eingriffe ${ }^{6}$ in die >Ordnung ‘ des Gebets eine gewisse Dynamik der Ablösung, Adaption und Zerlegung genuin christlicher Textgattungen konstatieren. Der Raum der auf den verschiedensten Genre-Ebenen auftretenden Gebetstexte reicht von konfessionell gebundener Literatur im Zeichen des Fortlebens religiöser Traditionen bis hin $\mathrm{zu}$ >freien Formen entweder affirmativ verhalten oder aber sich in einer negativ-kritischen Auseinandersetzung mit derselben niederschlagen. Die aus der letzteren spezifischen Referenz resultierenden Semantiken sind oftmals anti-christlich, atheistisch, macht- und kirchenkritisch.

Eben den doppelten Sachverhalt des Bezugs auf christliche Figuren bei gleichzeitiger Ablehnung jeder Form des institutionalisierten Glaubens versuche der von Georges Bataille in Anlehnung an Friedrich Nietzsche geprägte Begriff des »Hyperchristentums « analytisch zu beschreiben - eine These, für die sich der Germanist Clemens Pornschlegel in seinen Studien zur hyperchristlichen Struktur der Moderne starkmacht:

`Hyperchristentum` beschreibt also zunächst einmal den doppelten Sachverhalt des fortwährenden Bezugs auf christliche Bilder, Texte, Vorstellungen und Denkfiguren bei gleichzeitiger Ablehnung oder Überwindung jeder Form von >religiösem` Glauben und der entsprechenden moralischen Lebenspraxis. Indiziert ist mit dem Wort der freie, areligiöse, anti-institutionelle Gebrauch christlicher Schriften und theologischer Denkund Vorstellungsfiguren, der unmittelbar einhergeht mit der strikten Zurückweisung von kirchlicher Praxis, Sakramenten, Dogmen oder Moralschriften. ${ }^{7}$

Somit bringe der präfixierte Terminus (griechisch »hyper« = »oberhalb von, über etwas hinaus«) »die religiöse Signatur der westlichen Moderne ${ }^{8}$ am tref-

5 Aus kulturwissenschaftlicher Perspektive meint der hier neu eingeführte Begriff des Prekativs (spätlateinisch "precātīvus«, zu lateinisch »precārī» = »bitten«, »beten«) im weitesten Sinne alle kulturellen Manifestationen, die als `Bitte ( von X an Y gerichteter Wunsch) identifiziert werden können. Darunter einzuordnen sind neben religiösen Anbetungstexten auch die Formulare der Bürokratie sowie die Höflichkeitsformeln in der sozialen Kommunikation.

6 Assmann: Text und Kommentar (Anm. 4), 11.

7 Clemens Pornschlegel: Hyperchristen: Brecht, Malraux, Mallarmé, Brinkmann, Deleuze. Studien zur Präsenz religiöser Motive in der Moderne. Wien/Berlin 2011, 11.

8 Pornschlegel: Hyperchristen (Anm. 7), 173. 
fendsten zum Ausdruck, denn »das Christentum bleibt für alle Autoren - wie ironisch, polemisch, blasphemisch, parodistisch auch immer die christlichen Begriffe, Vorstellungen oder Textgattungen gehandhabt werden - ein zentraler Bezugspunkt. «"

Der vorliegende Beitrag rekurriert arbeitshypothetisch auf den Bataille'schen Begriff des Hyperchristentums und auf Pornschlegels Ausführungen in der Absicht, dieselben sowohl für die theoretische Rahmung als auch für die Argumentation fruchtbar zu machen.

Die Präsenz der religiösen Textgattung Gebet in außerreligiösen oder theologiefreien Kontexten der literarischen Moderne erscheint im Hinblick auf Verfahren der De- und Rekontextualisierung als besonders untersuchenswert. ${ }^{10}$ In historischer Hinsicht geht diese referentielle Besonderheit einerseits mit dem Bedeutungswandel des Gebets im Horizont der neuzeitlichen Religions- und Christentumskritik (Immanuel Kant, Friedrich Nietzsche) ${ }^{11}$ einher, andererseits steht das religiöse Prekativ angesichts der Weltkriegserfahrungen und der Shoah unter dem Vorzeichen der Krise und Unartikulierbarkeit. ${ }^{12}$ Als "poetogene Struktur ${ }^{13}$ ist das Gebet nicht nur als ein privilegiertes Medium der Austauschprozesse zwischen Religions- und Literatursystem lesbar ${ }^{14}$, sondern bietet sich als ein wichtiges zeitkritisches Reflexionsmedium der (post-)säkularen Moderne an.

Das breite Spektrum literarischer Prozeduren im Zeichen einer de- und rekontextualisierenden Referenz ließe sich - unter Vorbehalt - mit der politischen Gebetsumschreibung des radikalen Aufklärers Voltaire, Prière à Dieu (1763),

9 Pornschlegel: Hyperchristen (Anm. 7), 10.

10 Indem die Präsenz einer religiösen Gattung in der literarischen Postmoderne exemplarisch in den Blick genommen wird, reagiert der vorliegende Beitrag auf ein in der Forschung bereits formuliertes Desiderat einer Kulturgeschichte des Gebets (vgl. Andreas Kraß: Gebet. In: Klaus Weimar (Hg.): Reallexikon der deutschen Literaturwissenschaft, Bd. 1. Berlin/New York 1997, 662-664, hier 662).

11 Weiterführend dazu Friedrich Heiler: Das Gebet: eine religionsgeschichtliche und religionspsychologische Untersuchung. München ${ }^{3} 1921$, Hans Jürgen Luibl: Des Fremden Sprachgestalt. Beobachtungen zum Bedeutungswandel des Gebetes in der Geschichte der Neuzeit. Tübingen 1993, und Jürg Wüst-Lückl: Theologie des Gebetes. Forschungsbericht und systematisch-theologischer Ausblick. Fribourg 2007.

12 Weiterführend dazu Thomas Dienberg: Ihre Tränen sind wie Gebete. Das Gebet nach Auschwitz in Theologie und Literatur. Würzburg 1997.

13 Vgl. Stefan Keppler: Gebet als poetogene Struktur: systematische Aspekte, die Wissenskonfiguration um 1900 und Rilkes »Stundenbuch«. In: Rüdiger Zymner u. Manfred Engel (Hg.): Anthropologie der Literatur. Poetogene Strukturen und ästhetisch-soziale Handlungsfelder. Paderborn 2004, 338-355.

14 Vgl. Niklas Luhmann: Die Religion der Gesellschaft. Hg. v. André Kieserling. Frankfurt a. M. 2002, und ders.: Die Kunst der Gesellschaft. Frankfurt a. M. 1997; Heinz Schlaffer: Geistersprache. Zweck und Mittel der Lyrik. München 2012. 
öffnen und über zahlreiche Zwischenstationen weiterverfolgen. ${ }^{15}$ Um nur einige zu nennen: Eine unumgängliche, ja notwendige Zwischenstation wäre Friedrich Nietzsche, der kurz vor dem Anbruch des 20. Jahrhunderts rief: „Gott ist todt! Gott bleibt todt! Und wir haben ihn getödtet! Wie trösten wir uns, die Mörder aller Mörder? $\aleph^{16}$ Wenn Nietzsche die hohe Adresse der Anbetung (aus)löscht, stellt sich die Frage, welches diskursive Schicksal das Gebet im Denken dessen hat, der dachte, er stünde am Anfang eines Zeitalters des Atheismus. Es mag nicht überraschen: Es ist die Geste der antichristologischen Ablehnung, die sich an verschiedenen philosophischen Werkstationen (z. B. Der Antichrist, Also sprach Zarathustra etc.), aber auch an seiner Lyrik verfolgen lässt. Hinzu käme eine referentielle Formation, die ich » atheistisches Gebet« nennen möchte. Hugo Friedrich hat bereits gezeigt, dass die moderne Lyrik sich unter anderem paradoxer Strukturen bedient. ${ }^{17}$ Die Paradoxie des betenden Atheisten ließe sich an zahlreichen französischen Symbolisten (Charles Baudelaire, Jean-Arthur Rimbaud, Paul Verlaine etc.) oder an der Lyrik des spanischen Philosophen Miguel de Unamuno wie im Gedicht La oración del ateo in Rosario de sonetos líricos (1911) exemplifizieren. In den Phantastischen Gebeten des Dadaisten Richard Huelsenbeck begegnet man ferner einer Art von Referenz, die mit "primitiven" (im Sinne von "ursprünglichen") und vor-christlichen Bildern und Motiven arbeitet, während sich ımoneytheistischer Gebete wie 700 Intellektuelle beten einen Öltank an von Bertolt Brecht vor dem Hintergrund von Walter Benjamins These vom "Kapitalismus als Religion" untersuchen ließen. Zu schließen wäre die Reihe der Textanalysen womöglich mit dem ebenfalls politischen Gebet der russischen Frauenband Pussy Riot, dem musikalischen Punk-Gebet (2012).

Der Beitrag intendiert nicht das vermessene Unterfangen einer systematischen Kartierung dieses wenn auch zeitlich diffusen, in der europäischen Literatur jedoch weiten und prominent vertretenen Referenzfeldes "Gebet und literarische Moderne«, sondern richtet sich auf die stextnahe` Arbeit an einer der hier anzutreffenden Manifestationen: Im Werk Thomas Bernhards lassen sich die Rückgriffe auf und die Eingriffe in die >Ordnung des Gebets « vielerorts nachweisen. Sowohl in seinen lyrischen als auch in seinen narrativen Texten schöpft Bernhard aus dem christlich-katholischen Fundus kanonischer An-

15 Die negative Referenz auf das religiöse Phänomen des Gebets ist keineswegs ausschließlich ein Phänomen der säkularen (Post-)Moderne. Die zahlreichen Gebete und Gebetsparodien in der Antike boten immerhin Stoff für mehrere Forschungsarbeiten, vgl. Hermann Kleinknecht: Die Gebetsparodie in der Antike. Stuttgart 1937, und Wilhelm Horn: Gebet und Gebetsparodie in den Komödien des Aristophanes. Diss. Nürnberg 1970.

16 Friedrich Nietzsche: Die Fröhliche Wissenschaft. Kritische Gesamtausgabe. Hg. v. Giorgio Colli u. Mazzino Montinari. Bd. V/2, Berlin/New York 1973, hier 159.

17 Hugo Friedrich: Struktur der modernen Lyrik: von der Mitte des 19. bis zur Mitte des 20. Jahrhunderts. Hamburg 1967. 
betungsrhetorik. Dies geschieht jedoch nicht, um einen frommen Glaubensvollzug im Medium der Literatur zum Ausdruck zu bringen; vielmehr werden die Bezüge auf das Gebet in einer spezifischen und komplexen Weise gehandhabt. ${ }^{18}$

Im Mittelpunkt der Analyse steht die Gebetsumschreibung aus Bernhards Debütroman Frost (1963). Ihr literarischer Urheber ist Meister Strauch, ein Geistesmensch, der in der Eiseskälte eines Salzburger Gebirgsdorfs und inmitten von schwachsinnigen und verbrecherischen Landbewohnern lebt - eine der finstersten und zugleich hellsichtigsten Figuren der deutschsprachigen Literatur überhaupt.

Die von einem Famulanten im Zuge eines Beobachtungsauftrags aufgezeichneten maß- und schonungslosen Monologe des Malers nehmen den größten Teil des handlungsarmen Romans ein. Seine Wortkaskaden richten sich konsequenterweise und in sich steigernden Wiederholungen gegen Kirche und Katholizismus ebenso wie gegen Religion im Allgemeinen. Mitten im Roman (im

18 Die textimmanenten und diskursiven Bezüge auf das Gebet(-hafte), so häufig und komplex sie in den Texten Thomas Bernhards erscheinen, sind von der bisherigen Forschung nur peripher berücksichtigt worden, und zwar dann, wenn Forschungsfragen nach Lektüremodellen im signaturhaften, liturgischen Gebrauch (Juliane Vogel: Die Gebetbücher der Philosophen: Lektüren in den Romanen Thomas Bernhards. In: Modern Austrian Literature 21 [1988], 173-186), nach dem Gottesbegriff, dem Glaubensvollzug bzw. dem Verlust transzendentalen Glaubens (Adolf Holl: Thomas Bernhard und die Religion. In: Pia Janke u. Ilija Dürrhammer (Hg.): Der »Heimatdichter" Thomas Bernhard. Wien 1999, 209-215; Cornelius Hell: "Geheiligt werde kein Name«. Religionsverlust und Gottesvernichtung im Werk Thomas Bernhards. In: Peter Tschungall (Hg.): Perspektiven einer Begegnung am Beginn eines neuen Milleniums. Religion, Literatur, Künste. Salzburg 2001, 376-386, und ders.: Zensierte Kampfgebete. Neues Licht auf Thomas Bernhards Verhältnis zur Religion. In: Orientierung 68 (2004), 43-49; Heinrich Schmidinger: "Gott« im Werk Thomas Bernhards. In: Manfred Mittermayer u. Martin Huber (Hg.): Thomas Bernhard Jahrbuch. Bd. 2. Wien u. a. 2003, 35-66; Jan Süselbeck: Das Gelächter der Atheisten: Zeitkritik bei Arno Schmidt und Thomas Bernhard. Frankfurt a. M. 2006; Gregor Thuswaldner: De Deo abscondito. Religiöse Konflikte bei Thomas Bernhard. In: Olaf Berwald u. ders.(Hg.): Der untote Gott. Religion und Ästhetik in der deutschen und österreichischen Literatur des 20. Jahrhunderts. Köln 2007, 159-176), nach Negation(sprozessen) (Manfred Mixner: Vom Leben zum Tode. Die Einleitung des Negations-Prozesses im Frühwerk von Thomas Bernhard. In: Manfred Jurgensen (Hg.): Bernhard Annäherungen. Bern/München 1981, 65-97; Philipp Schönthaler: Negative Poetik. Die Figur des Erzählers bei Thomas Bernhard, W. G. Sebald und Imre Kertész. Bielefeld 2011) oder Fragen nach werkkonstitutiven Strukturen wie Litanei, Wiederholung, Auslöschung oder nach Sprachrhetorik vordergründig waren (Oliver Jahraus: Die Wiederholung als werkkonstitutives Prinzip im CEuvre Thomas Bernhards. Frankfurt a. M. u. a. 1991; Wendelin Schmidt-Dengler: Das Gebet in die Sprache nehmen: zum Säkularisationssyndrom in der österreichischen Literatur der siebziger Jahre. In: Christiane Pankow (Hg.): Österreich - Beiträge über Sprache und Literatur. Umeå 1992, 45-62; Christoph Kappes: Schreibgebärden. Zur Poetik und Sprache bei Thomas Bernhard, Peter Handke und Botho Strauss. Würzburg 2006, oder Joachim Knappe u. Olaf Kramer (Hg.): Rhetorik und Sprachkunst bei Thomas Bernhard. Würzburg 2011). 
Kapitel »Siebzehnter Tag») führt der Kunstmaler Strauch dem erzählenden Medizinstudenten seine "Komödientragödie« vor: " 'Sehen Sie`, sagte er, `dieser Baum tritt auf und sagt, was zu sagen ich ihm aufgetragen habe, irgendwann aufgetragen habe, einen Vers, einen unverständlichen, die Welt auf den Kopf stellenden Vers, einen sogenannten Antigottesvers, verstehen Sie! « ${ }^{19}$

Die Passage antizipiert jene Gebetskontrafaktur, die im zweiten Abschnitt des Kapitels »Neunzehnter Tag" völlig unvermittelt formuliert wird und die in der bisherigen Forschung Charakterisierungen fand wie »eine blasphemische Travestie ${ }^{20}$, »eine ganz auf Provokation abgestellte Fassung des Vaterunsers ${ }^{21}$ oder ein »blasphemischer Rückverweis « ${ }^{22}$ auf die christliche Bezugsebene etc. Es handelt sich hier um ein 'pervertiertes`, ins Gegenteil verkehrtes Gebet, dessen Erschließung in einem ersten Anlauf anhand der Unterschiede zu dem orthodoxen Gebetstext des biblischen Kanons erfolgen kann. ${ }^{23}$ Beide Texte seien hier gegenübergestellt:

(Prätext)

Vater unser im Himmel geheiligt werde dein Name.

Dein Reich komme.

Dein Wille geschehe, wie im Himmel, so auf Erden. Unser tägliches Brot gib uns heute. Und vergib uns unsere Schuld, wie auch wir vergeben unsern Schuldigern. Wie auch wir vergeben keinen Schuldigern.

19 Thomas Bernhard: Frost. Werke. Hg. v. Martin Huber u. Wendelin Schmidt-Dengler. Bd. 1. Hg. v. dens. Frankfurt a. M. 2003, 200.

20 Paola Bozzi: »Das Wort des Todes«. Thomas Bernhard und Charles Péguy. In: Joachim Hoell u. Kai Luehrs-Kaiser (Hg.): Thomas Bernhard. Traditionen und Trabanten. Würzburg 1999, 151-158, hier 156.

21 Erich Jooß: Aspekte der Beziehungslosigkeit. Zum Werk von Thomas Bernhard, München 1976.

22 Manfred Mittermayer: Thomas Bernhard. Frankfurt a. M. 2006, 78.

23 Beim kanonischen Vaterunser handelt es sich um das einzige Gebet der christlichen Gemeinde, das im Neuen Testament (in zwei leicht verschiedenen Versionen in Mt 6, 9-15 und Lk 11, 2-4) von Jesus Christus gegeben und darüber hinaus mit einer Gebetsaufforderung (»Darum sollt ihr so beten [...]«, Mt 6,9a) versehen wird. Nicht zuletzt werden die eingangs dieses Beitrags thetisch formulierten redaktionellen Tabus in einem Interview des Kirchenhistorikers Christoph Markschies vom 12.06.2010 bestätigt, der in Deutschlandradio Kultur - Religionen die kleinen Änderungen am Vaterunser-Text in den neueren Bibelübersetzungen in einem halb jovialen Ton kommentierte: "Also erst mal würd ich davor warnen, das Vaterunser umzuschreiben, denn mindestens gibt es gute Gründe anzunehmen, dass uns Jesus von Nazareth dieses Gebet lehrt." Uwe Birnstein: Der Gott, der in Versuchung führt. Muss das Vaterunser geändert werden? Online unter: www. deutschlandradiokultur.de/der-gott-der-in-versuchung-fuehrt.1278.de.html?dram:artic le_id=192552 (08.09.2014). 
Und führe uns nicht in Versuchung, sondern erlöse uns von dem Bösen. Amen.
Führe uns in Versuchung

Und erlöse uns von keinem Übel. Amen.

So geht es ja auch. ${ }^{24}$

Aus der Gegenüberstellung wird deutlich, dass die redaktionellen Eingriffe auf unterschiedlichen Niveaus stattfinden - als grammatische und logische Verneinung - und dass sie somit gegen die eingangs beobachtete normative Fixierung des Gebets im Wortlaut und im Umfang gerichtet sind.

Vordergründig ist eine (fast) durchgängige Prozedur zu beobachten: Was der theologische Kanon affirmativ formuliert, wird bei Bernhard negativ umformuliert, oder anders betrachtet: eine primäre Bedeutung wird negiert, um eine gegenläufige aufzubauen. Darauf wird unten zurückzukommen sein, vorab sei jedoch festgehalten, dass diese Prozedur einer formallogischen Umwertung wie "p wird zu $\neg \mathrm{p}$ « (und vice versa) ${ }^{25}$ entspricht. Dafür schlage ich hier den Begriff Umpolung vor. Das literarische Vaterunser (Folgetext) baut punktuell auf dem kanonischen Vaterunser (Prätext) auf; der Folgetext ist in seiner intertextuellen Organisation Aneignung, Absorption und Transformation des Textes, aus dem er hervorgeht. Die für Thomas Bernhard so charakteristische formal-ästhetische Qualität der Wiederholung kann man in der metatextuellen Struktur des `neuen Gebets rasch erkennen.

Welche interpretatorischen Konsequenzen zieht ein solcher auf den ersten Blick einfacher Kunstgriff der Negativierung mit sich? Eine komparative Perspektivierung religionskanonischer vs. literarischer Konventionen sowie das Heranziehen der Intertextualität als Analyseinstrument können dabei helfen, Referenzen aufzuspüren und somit das Problembewusstsein für die Bedeutung der Bernhard'schen Autorintention zu schärfen.

In Analogie zu einem in Raum und Zeit definierten Objekt beinhaltet der erste Vers beider Texte eine Ortszuweisung: Im kanonischen Vaterunser deutet der Zusatz der Anrede auf den Himmel, der in der pragmatischen Theologie als regnum Dei gilt. ${ }^{26}$ Bei Bernhard findet eine Umpolung auf der Vertikalen statt, denn die poetische Sicht operiert hier mit Antipoden. Der Zusatz der Anrede ist nicht »im Himmel«, sondern »in der Hölle«, also am entgegengesetzten Punkt religiöser Erfahrung, am »Ort der äußersten Gottesferne«. ${ }^{27}$

24 Bernhard: Frost (Anm. 19), 221.

25 Vgl. Thomas Zoglauer: Einführung in die formale Logik für Philosophen. Göttingen 2008.

26 Zur pragmatischen Theologie des Vaterunser im Allgemeinen: Richard Mössinger. Zur Lehre des christlichen Gebets. Göttingen 1986, Georg Nicolaus: Die pragmatische Theologie des Vaterunsers und ihre Rekonstruktion durch Martin Luther. Leipzig 2005, oder Jürgen Werbick: Vater unser. Theologische Meditationen zur Einführung ins Christsein. Freiburg i. Br. u. a. 2011.

27 Schmidinger: »Gott« im Werk Thomas Bernhards (Anm. 18), 37. Das hier eröffnete Anti- 
Die negative Formel »Geheiligt werde kein Name« zeigt - allein genommen eine gewisse Nähe zu Schweigeordnungen der negativen Theologie (Namenstabu). ${ }^{28}$ Nichtdestotrotz lässt sich die Trias "Geheiligt werde kein Name« "Zukomme uns kein Reich« - »Kein Wille geschehe« über die mitschwingende Verweigerung der religiösen Heilsgeschichte hinaus vielmehr als Anspielung auf Elemente nationalsozialistischer Sprachpraxis interpretieren, etwa die Struktur des Hitlergrußes (Interjektion Heil gefolgt vom Namen), die Bezeichnung für den nationalsozialistischen deutschen Staat (Drittes Reich) sowie die ubiquitäre "Wille-des-Führers«-Rhetorik der nationalsozialistischen Gesetzgebung, ${ }^{29}$ zumal Strauch in seinen Tiraden oftmals auf die "Kriegszeit" und die Auswirkungen des Krieges verweist. ${ }^{30}$

Das Umschreiben von optativen Formeln in deprekative Forderungen wird auch in den nächsten Zeilen fortgesetzt, wobei die Mehrdeutigkeit von »Schuld» und "Schuldigern" bzw. die Ununterscheidbarkeit von debitum und peccatum im Deutschen die Interpretation erschwert. Das negative Sprachspiel Strauchs ist nicht auf Vergebung und Erlass (einer Schuld oder einer Strafe) ausgerichtet, sondern im Gegenteil auf die Nicht-Tilgung, quasi als Memento an die begangenen Verfehlungen und Missetaten im Allgemeinen und an die Verbrechen des Nationalsozialismus im Besonderen.

Auch die letzten Bitten werden nach dem Verfahren der Umpolung umgeschrieben und steigern die Negationsperspektive als Verweigerung jeglicher Absolution. Trotzdem trifft die umschreibende Prozedur nicht gänzlich auf den

poden-Verhältnis erinnert an Bernhards frühe Lyrik: »Warum muss ich die Hölle sehen? Gibt es keinen anderen Weg / zu Gott? / Eine Stimme: Es gibt keinen anderen Weg! Und dieser Weg / Führt über den Tag der Gesichter, / er führt durch die Hölle.« (Thomas Bernhard: Der Tag der Gesichter. Gesammelte Gedichte. Frankfurt a. M. 1993, 11.)

28 Seit seinen systematischen Anfängen in der Spätantike hat das Denken der negativen Theologie die Grenze des Wissens als Nichterkennbarkeit der göttlichen Transzendenz bestimmt und diesen äußersten Horizont der Erkenntnis als eine (sprachliche) Grenze der Kommunizierbarkeit und Artikulierbarkeit verstanden: Das Absolute ist dementsprechend unnennbar, unsagbar, unbestimmbar. Philosophen und Theologen haben diese Grenze der Sprache in einer Sprache der Grenze zu reflektieren versucht, jedes Reden von `Gott muss negativ und apophatisch (gr. »nicht aussprechbar«) bleiben. Dazu Dirk Westerkamp: Via negativa. Sprache und Methode der negativen Theologie. München 2006.

29 Der preußische Ministerpräsident Hermann Göring proklamierte in einer Rede vor hohen Staatsanwälten im Sommer 1934: „Das Recht und der Wille des Führers sind eins!« (Völkischer Beobachter, 14.07.1934). In: Walther Hofer: Der Nationalsozialismus. Dokumente 1933-1945. Frankfurt a. M. 2004, 77. Im NS-Staat wurden die Willensbekundungen des Führers als juristisch bindend betrachtet. Vgl. Hubert Schorn: Die Gesetzgebung des Nationalsozialismus als Mittel der Machtpolitik. Frankfurt a. M. 1963.

30 Im Hinblick auf die verallgemeinernde Umschreibung ohne konkrete Benennung von Verbreche(r)n des Nationalsozialismus in den Monologen des Malers Strauch vgl. Josef Mautner: Nichts Endgültiges. Literatur und Religion in der späten Moderne. Würzburg 2008, $110 \mathrm{f}$. 
neuen Text zu. Vielmehr partizipieren Anfang und Ende des Anti-Gebets durch die Beibehaltung des Adressaten »Vater unser « und der abschließenden Formel "Amen« am kanonischen Gebetstext. Das `neue` Gebet wird außerdem doppelt sanktioniert: zunächst durch das orthopraktische "Amen« mit der Funktion einer Wahrheit beanspruchenden Schlussformel ${ }^{31}$ und anschließend durch ein parenthetisches "So geht es ja auch", das zugleich relativiert und pluralisiert.

In diesem letzteren Zusammenhang drängen sich mehrere Fragen auf: Wenn der religiöse Bezugspunkt `haften ‘ bleibt und als `Spur ` wiedererkennbar ist, wie ist die Beibehaltung des Adressaten "Vater unser" trotz des im Übrigen umpolenden Eingriffs auf der Ebene der im Imperativ formulierten Bitten zu interpretieren? Lässt die Erweiterung der liturgischen Formel um eine profane Sanktion das gegenläufige Gebet eher als Fortgang unter verändertem Vorzeichen oder als Indiz einer atheistischen Zäsur auftreten? Welche Rolle spielt der Wahrheitsbegriff und wie ist Strauchs adversative Referenz auf den Wahrheitsgehalt des Gebets zu verstehen vor dem Hintergrund der Aussagen Thomas Bernhards zum Thema >Wahrheit im Allgemeinen? Wenn auch eine erschöpfende Behandlung des Fragenkomplexes in diesem Rahmen nicht möglich ist, soll im Folgenden etwas näher darauf eingegangen werden.

Begonnen sei mit der Frage nach der beibehaltenen Adresse »Vater unser«. In seinem Vortrag Wie nicht sprechen. Verneinungen (1987) hinterfragt Jacques Derrida die Zitierbarkeit des Gebets und reflektiert über die Apostrophe als Bestimmung des Adressaten im Gebet. Derridas Gedanken lassen sich in diesem Zusammenhang als Kontextualisierungsangebot an Thomas Bernhard herantragen. An den vielfältigen Erfahrungen und Bestimmungen dessen, was man als `Gebet` bezeichnet, hebt Derrida das Moment der Adresse als Bezug zu einer Präsenz im Anderswo und als Geste der Anerkennung hervor:

Es müsste in jedem Gebet eine Adresse an den anderen als den anderen geben und ich möchte sagen, auf die Gefahr hin zu schockieren, Gott zum Beispiel. Als Akt, sich an den anderen als den anderen zu adressieren, muss er gewiss beten (prier), das heißt bitten (demander), anflehen, ersuchen. Worum, das zählt wenig, und das reine Gebet bittet den anderen um nichts anderes als es anzuhören, es anzunehmen, für es gegenwärtig zu sein $[\ldots] .^{32}$

Ferner befragt Derrida den Themenkomplex Gebet, indem er ihn explizit ähnlich wie Thomas Bernhard - mit den Philosophemen >Negation >Wahrheit in Zusammenhang bringt und sich an folgende Stelle aus Aristoteles' Peri hermeneia heranarbeitet:

31 Vgl. Ulrich Heckel: Der Segen im Neuen Testament. Begriffe, Formeln, Gesten; mit einem praktisch theologischen Ausblick. Tübingen 2002, hier besonders Kapitel 3.3.7 Das Amen, 308-312.

32 Jacques Derrida: Wie nicht sprechen. Verneinungen. Hg. v. Peter Engelmann. Wien 1989, 76. 
Jedes Wortgefüge hat zwar eine Bedeutung [...], ein Behauptungssatz ist aber nicht jedes, sondern nur eines, dem es zukommt, wahr oder falsch zu sein. Nicht allen kommt dies zu. So ist zum Beispiel eine Bitte zwar ein Wortgefüge, aber weder wahr noch falsch. ${ }^{33}$

Trotz aller Knappheit wird uns hier eine Definition überliefert, die das Fehlen jeglichen Bezugs auf die Wahrheitswerte "wahr" oder "falsch» zum Hauptmerkmal des Gebets avanciert. Diese logische Figur des Dritten (»weder wahr noch falsch«) macht Derrida zufolge deutlich, dass die Wahrheit des Gebets »eine Überwahrheit einer Überwesentlichkeit ist, denn es nennt und ruft, was >ist ` und so wie es 〉ist ` jenseits des Seins. ${ }^{34}$ Noch wichtiger erscheint mir im Kontext des literarischen Anti-Gebets Derridas Unterscheidung zwischen Gebet (eukhé) und Lobpreisung (hymnein) nach dem Kriterium des propositionalen Gehalts (als Aussage über): Selbst wenn sie keine prädikative Affirmation geläufigen Typus ist, so wahrt die Lobpreisung die Struktur einer Prädikation, einer sprachlichen Handlung, die etwas von einem Gegenstand sagt bzw. durch die einem Gegenstand Eigenschaften zu- oder abgesprochen werden. Das Gebet hingegen apostrophiert, adressiert sich also an einen anderen und in dieser reinen Bewegung bleibt es - so Derrida - absolut ante-prädikativ. ${ }^{35}$

Was die Apostrophe und die Prädikation bei Bernhard anbelangt, so ist Strauchs Gebet ein literarisches Sprachspiel mit dem propositionalen Gehalt des Glaubens. Nicht bloß Destruktion affirmativer Theologeme im Modus atheistischer Anti-Prädikation ist hier am Werk, sondern Repetition religiöser Ritualsprache. Selbst wenn ein solches Verfahren im Paradox endet, scheint es logisch zwingende Gründe zu geben, lieber beide Seiten des Paradoxes zusammenzuhalten, als sie auseinanderfallen zu lassen und sich auf Gedeih und Verderb auf eine der beiden Seiten festzulegen. Dies zeigt sich nicht zuletzt in der relativierenden und pluralisierenden Formulierung "So geht es ja auch", die das kanonische Gebet in seiner Gültigkeit einschränkt und in einem übergeordneten

33 Aristoteles: Peri hermeneias. Werke in deutscher Übersetzung. Bd. 1, Teil 2. Übers. u. erl. v. Hermann Weidemann. Darmstadt 1994, hier 6. Zu beachten ist, dass die griechischen Vo-

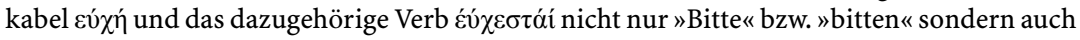
"Gebet« und »beten« im förmlich-rituellen Sinne bedeuten. Ohne auf die philologischen

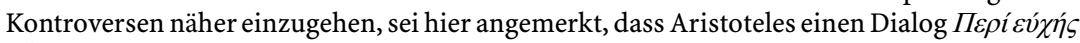
(Über das Gebet) geschrieben hat, wie aus den Schriftenverzeichnissen des Diogenes Laertios hervorgeht. Das einzige erhaltene Fragment verrät jedoch nichts über Aufbau und Inhalt des Dialogs: "Dass Aristoteles etwas im Blick hat, was über das Denken und über das Sein hinausgeht, sagt er deutlich gegen Ende des Buches Über das Gebet: `Gott ist entweder Denken oder noch etwas jenseits des Denkensı. (Simplikios: In Aristotelis de caelo commentaria, II 12, 282 b 10. In: Commentaria in Aristotelem Graeca Bd. 7. Hg. v. Johan L. Heiberg. Berlin 1894, 485; hier zitiert nach Aristoteles: Fragmente zu Philosophie, Rhetorik, Poetik, Dichtung. Hg. v. Hellmut Flashar. Darmstadt 2006, 50).

34 Derrida: Wie nicht sprechen (Anm. 32), 76.

35 Derrida: Wie nicht sprechen (Anm. 32), $75 \mathrm{f}$. 
Zusammenhang betrachtet. Dennoch ist es wichtig zu erkennen, dass die Abtönungspartikel $\mathrm{ja}^{36}$ (wie das ähnlich gebrauchte aber) den Indikator für eine adversative Rede darstellt, die für Bernhards Sprachgebrauch insgesamt kennzeichnend ist und die jenem sprachlich fundierten Wahrheitsbegriff entspricht, den Bernhard beispielhaft in einem Interview aus dem Jahre 1975 zum Ausdruck bringt:

Aber es muss immer Leute geben, die immer ein Aber sagen. Es gibt immer nur Leute, die alle Sachen bestimmen. Aber es bleibt alles ohne Aber. Die Wahrheit ist im Grunde nur, dass man Aber dranhängt und den Satz vollendet. [...] Aber eine Schönheit ohne das Aber ist ein reiner Unsinn, eine Verfälschung. ${ }^{37}$

Ohne auf die Syntax und die Semantik des Ja und des Aber genauer einzugehen, sei hier angemerkt, dass die adversative Referenz auf das christliche Zentralgebet im Zeichen einer philosophischen Grammatik realisiert wird, die Satz und Gegensatz kontrastiert und zugleich koordiniert. Vor diesem Hintergrund lässt die Erweiterung der liturgischen Formel um eine umgangssprachliche Sanktion das gegenläufige Gebet nicht als (atheistische) Zäsur, sondern als Kontinuität und Schließung des Problems auftreten.

Auch weitere Textstellen im Roman bieten Anlass zu ähnlichen mikrologischen Beobachtungen, die m. E. interessante Tiefendimensionen der adversativen Referenz zugänglich machen. Zum Beispiel: Die Frage an den Famulanten ")Glauben Sie an Christus? " $^{38}$ erwartet keine Antwort; stattdessen formuliert der Maler scharf zugespitzte und wuchtige Sentenzen:

»Alles Gesagte ist Unsinn. Die Religionen täuschen darüber weg, daß alles Unsinn ist, wissen Sie. Das Christentum ist Unsinn. Ja. Als Christentum. Die Gebetswelt, das sind Zustände, die alles falsch wiedergeben. Die alles zu nichts machen. Gerade die Gebetswelten! Das ist wahr.« Aber der Mensch lebe gern falsch und unter falschen Eindrücken, »die seinen Kopf auf den Boden herunterdrücken.«" ${ }^{39}$

An anderer Stelle erhebt der Maler drohend die Faust gegen die Monstranz, ${ }^{40} \mathrm{um}$ dann zu den folgenden, nicht minder radikalen Urteilen zu gelangen:

"Sehen Sie mein Theater? Sehen Sie das Theater der Furchtsamkeit? Das Theater der Unselbständigkeit Gottes? Welchen Gottes?« Er drehte sich um und sagte: „Gott ist eine

36 Weiterführend siehe Elke Hentschel: Funktion und Geschichte deutscher Partikeln: ja, doch, halt und eben. Tübingen 1986, und Harald Weydt: Abtönungspartikel. Bad Homburg v. d. H. 1969.

37 Thomas Bernhard: Der Wahrheit auf der Spur. Reden, Leserbriefe, Interviews, Feuilletons. Hg. v. Wolfram Bayer u. a. Berlin 2011, 101.

38 Bernhard: Frost (Anm. 19), 117.

39 Bernhard: Frost (Anm. 19), 175.

40 Bernhard: Frost (Anm. 19), 134. 
einzige große Verlegenheit! Eine ungeheure Verlegenheit der Gestirne. Aber«, sagte er, legte den Zeigefinger an den Mund: „Wir wollen darüber schweigen. ${ }^{41}$

Sätze wie »>Das Christentum ist Unsinn. Ja. Als Christentum « oder " die Kirche hat ja, wie sie auch ist, gar keine Existenzberechtigung. Wenigstens nicht als Kirche « ${ }^{42}$ sind trotz ihrer vordergründigen Einfachheit nicht ganz unproblematisch. Die Deutungsschwierigkeit rührt daher, dass der Bezugsreferent und der als-Adjunkt identisch sind. Eine Kreisbewegung, bei der die Spezifikation auf den Referenzpunkt zurückverweist. Womöglich verringert sich die Deutungsschwierigkeit, wenn man die als-Phrasen auf der werkinternen Ebene im Zusammenhang mit dem »Antigottesvers «, mit der direkten Frage » Glauben Sie an Christus?« und mit den Reflexionen des Famulanten über "das Außerfleischliche« auf der Eröffnungsseite des Romans liest:

Eine Famulatur muß auch mit außerfleischlichen Tatsachen und Möglichkeiten rechnen. Mein Auftrag, den Maler Strauch zu beobachten, zwingt mich, mich mit solchen außerfleischlichen Tatsachen und Möglichkeiten auseinanderzusetzen. Etwas Unerforschliches zu erforschen. Es bis zu einem gewissen erstaunlichen Grad von Möglichkeiten aufzudecken. Wie man eine Verschwörung aufdeckt. Und es kann sein, daß das Außerfleischliche, ich meine damit nicht die Seele, daß das, was außerfleischlich ist, ohne die Seele zu sein, von der ich ja nicht weiß, ob es sie gibt, von der ich aber erwarte, daß es sie gibt, daß diese jahrtausendealte Vermutung jahrtausendealte Wahrheit ist; es kann durchaus sein, daß das Außerfleischliche, nämlich das ohne die Zellen, das ist, woraus alles existiert, und nicht umgekehrt und nicht nur eines aus dem andern. ${ }^{43}$

Vergegenwärtigt man sich zudem - auf der werkexternen Ebene - die in der ökumenischen Dogmatik so zentrale Theorie der Inkarnation bzw. der Fleischwerdung oder Menschwerdung Christi ${ }^{44}$ - die Thomas Bernhard vermutlich spätestens aus der Zeit im katholischen Internat kannte -, dann lassen sich die Sentenzen des Malers Strauch in neuem Licht interpretieren:

Man darf sich fragen, ob Strauchs Vaterunser auf eine subtile Art und Weise der Versuch einer Annäherung an religiöse Inhalte durch die Stilisierung derselben ist oder gar das diskrete Unternehmen, eine neue Theologie zu errichten? Diese Fragen sind m. E. negativ zu beantworten. Der Maler Strauch ist ein Dekonstrukteur, dessen kritisches Hinterfragen alles überprüft, zersetzt und, wie gezeigt wurde, eventuell neu zusammensetzt. Seine Triebkraft ist die der hyperchristlichen Revolte gegen jede religiös legitimierte Knechtschaft, denn trotz der im Roman spürbaren Überwindungs- und Ablösungsbewegungen ver-

41 Bernhard: Frost (Anm. 19), 201 (Hervorhebung im Original).

42 Bernhard: Frost (Anm. 19), 222 (Hervorhebung im Original).

43 Bernhard: Frost (Anm. 19), 7.

44 Edmund Schlink: Schriften zu Ökumene und Bekenntnis. Bd. 2: Ökumenische Dogmatik. Göttingen 2003, besonders 276-288. 
schwindet das religiöse Referenzsystem nicht. >Dekonstruieren ‘ heißt allerdings nicht `destruieren`, und für Bernhards Geistesmenschen heißt es umso weniger, den christlichen Gott durch die Vernunft des Atheisten zu substituieren. Ganz im Gegenteil: Strauchs mitunter irritierendes Zersetzungsmanöver unterscheidet sich von einer bloßen Kritik dadurch, dass es auf selbstdekonstruktive Momente abzielt, die das Christentum selbst als Religion und Institution in sich birgt und mitschleppt. Nochmals: "»Die Gebetswelt, das sind Zustände, die alles falsch wiedergeben. Die alles zu nichts machen. Gerade die Gebetswelten! « ${ }^{45}$ Bedeutet die ,falsche Wiedergabe etwa, die Transzendenz ins "Fleischliche« herunterzuziehen, also ins Messbare, Vergleichbare, Wägbare? Wenngleich eine interpretatorische Festlegung unmöglich scheint - geschuldet durch eine in Strauchs Monologen vorzufindende sdeutliche Undeutlichkeit` als kontrolliertes Offenlassen der Sachverhalte -, darf hier festgehalten werden: Weit überwiegend ist die rhetorische Geste der Außerkraftsetzung religiöser und dogmatischen Mächte und im Umkehrschluss der Entlarvung anti-religiöser und a-theistischer Züge im »Christentum [Christi] « ${ }^{46}$ als (Kirchen-)Religion.

Texte können in unterschiedlicher Weise auf die jeweiligen Prätexte reagieren. Die Literaturtheoretikerin Renate Lachmann unterscheidet drei Modelle intertextuellen Schreibens:

Partizipation schließt im Wiederholen und Erinnern der vergangenen Texte ein Konzept ihrer Nachahmung ein. >Tropikı [...] im Sinne des Tropus-Begriffs Harold Blooms als Wegwenden des Vorläufertextes, als Kampf, tragischer Kampf gegen die sich in den eigenen Text notwendig einschreibenden fremden Texte, als Versuch der Überbietung, Abwehr und Löschung der Spuren des Vorläufertextes; Transformation dagegen als eine über Distanz, Souveranität und zugleich usurpierende Gesten sich vollziehende Aneignung des fremden Textes, die diesen verbirgt, verschleiert, mit ihm spielt und durch komplizierte Verfahren unkenntlich macht, respektlos umpolt, viele Texte mischt $[\ldots]^{47}$

Man kann Bernhards `hyperchristliches Gebet` als Beispiel für einen Text lesen, der in einem zugleich partizipativen, tropischen und transformativen Referenzmodus seine Bedeutung aufbaut, auch wenn es schwierig zu sagen ist, was Strauchs `Gebet bedeutet: Ein Wiederschreiben der Apostrophe, ein Widerschreiben der Negativierung und Relativierung und ein Umschreiben religiöser Energien - dies alles zeugt von der widerständigen Kreativität des literarischen Hyperchristen.

45 Thomas Bernhard: Frost (Anm. 19), 175.

46 Friedrich Nietzsche: Der Antichrist. Sämtliche Werke. Kritische Ausgabe in 15 Bänden. Hg. v. Giorgio Colli u. Mazzino Montinari. Bd. 6. München 1980, 197.

47 Renate Lachmann: Gedächtnis und Literatur. Intertextualität in der russischen Moderne. Frankfurt a. M. 1990, 39. An einer anderen Stelle (ebd., 38) bringt Lachmann die drei Modelle auf die anschauliche Formel: Partizipation als "Weiterschreiben und Wiederschreiben", Tropik als »Widerschreiben« und Transformation als »Umschreiben«. 